\title{
EFECTO DE LA DENSIDAD DE PLANTACIÓN INICIAL EN EL MÓDULO DE ELASTICIDAD DINÁMICO DE ÁRBOLES EN PIE Y TROZAS DE UNA PLANTACIÓN DE PINO RADIATA DE 28 AÑOS, EN LA ZONA DE ARENALES, CHILE
}

\section{EFFECT OF INITIAL PLANTING DENSITY IN DYNAMIC MODULUS OF ELASTICITY IN STANDING TREES AND LOGS OF 28 YEARS OLD RADIATA PINE PLANTATION IN SANDY SOIL, CHILE}

\author{
L. Soto ${ }^{1}$, L. Valenzuela ${ }^{2}$, J.P. Lasserre ${ }^{1}$
}

\section{RESUMEN}

En un ensayo de densidad de plantación inicial (DPI), se evaluó el efecto de cuatro tratamientos $\left(2500,1667,833\right.$ y 625 árb·ha $\left.{ }^{-1}\right)$ en el módulo de elasticidad dinámico $\left(\mathrm{MOE}_{d}\right)$ de Pinus radiata $\mathrm{D}$. Don, de 28 años, creciendo sobre un suelo de la serie Arenales, en la Región del Biobío, Chile.

El $\mathrm{MOE}_{d}$ se determinó con tecnología acústica utilizando el método de tiempo de vuelo (Tv) para árboles en pie y posterior a su volteo se usó el método de resonancia (Res) para dos trozas de $5 \mathrm{~m}$ de longitud (primera y segunda troza), extraídas desde la base y a lo largo del fuste de cada árbol.

La DPI no afectó significativamente el $\mathrm{MOE}_{d}$ de los tratamientos más densos (2500, 1667 y 833 árb $\left.\cdot \mathrm{ha}^{-1}\right)$, tanto para árboles en pie, como para las dos trozas. En cambio, el tratamiento menos denso (625 árb·ha ${ }^{-1}$ ) presentó el mayor $\mathrm{MOE}_{d}$, significativamente diferente en árboles en pie y la primera troza, y similar entre la primera y segunda troza.

Palabras clave: Módulo de elasticidad dinámico, Densidad de plantación inicial, Pinus radiata.

\begin{abstract}
The influence of initial planting density (DPI) on the dynamic modulus of elasticity $\left(\mathrm{MOE}_{d}\right)$ was examinated at a 28 years old Pinus radiata D. Don spacing experiment with four treatments (2500, 1667, 833 and 625 stem $\cdot$ ha $^{-1}$ ) growing on sandy soil, in the Biobío Region, Chile.

The $\mathrm{MOE}_{d}$ acoustic technology was determined using the method of time of flying (Tv) for standing trees and the resonance method (Res) for two logs of $5 \mathrm{~m}$ long, extracted from the tree base to the top of each tree.

$\mathrm{MOE}_{d}$ was not significantly influenced by DPI in high initial stocking treatments (2500, 1667 and $833 \mathrm{stem} \cdot \mathrm{ha}^{-1}$ ), both standing trees and logs level. In contrast, the lowest initial stocking treatment $\left(625 \mathrm{stem} \cdot \mathrm{ha}^{-1}\right.$ ) had the highest $\mathrm{MOE}_{d}$, significantly different in standing trees and the first the log, and similar between the first and second log.
\end{abstract}

Keywords: Dynamic modulus of elasticity, initial planting density, Pinus radiata.

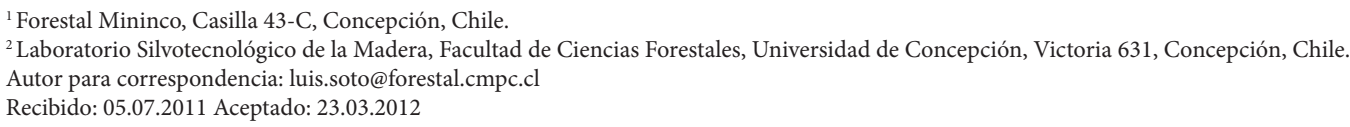




\section{INTRODUCCIÓN}

La especie Pinus radiata se ha posicionado como la especie forestal más importante en la economía del sector forestal chileno, reflejado tanto en la extensión de sus plantaciones, como en sus procesos industriales y comerciales. Su madera, como materia prima industrial, presenta una calidad variable debido a que las propiedades y características de su madera, varían dentro del mismo fuste de un árbol (Valenzuela y Nakayama 1991a, b), así como también, para fustes de árboles creciendo en diferentes localidades geográficas de Chile, donde esta especie ha sido introducida.

El uso eficiente de la madera de Pinus radiata, como material estructural, no está exento de esta variabilidad, ya que el módulo de elasticidad en flexión (MOE), la propiedad mecánica más importante para estimar la calidad de la madera estructural (Walford 1985), está asociada a la rigidez del material. Por lo tanto, madera con un alto MOE es un material más rígido y, por ende, más difícil de flectar (Roth et al. 2007), de esta forma, altos valores de MOE significan mejor calidad de la madera para fines estructurales.

En la madera, se pueden determinar dos tipos de $\mathrm{MOE}$, uno estático $\left(\mathrm{MOE}_{e}\right)$ y otro dinámico $\left(\mathrm{MOE}_{d}\right)$. Básicamente, en el primero se utiliza un dispositivo que permite aplicar una carga, registrar una deflexión dentro del rango elástico, y con las dimensiones del elemento de madera se obtiene el $\mathrm{MOE}_{e}$, y en el segundo, se usan dispositivos que facilitan el viaje de ondas acústicas a través del elemento de madera y registran el tiempo o la velocidad de tránsito de estas ondas, que en conjunto con la densidad de la madera permiten obtener el $\mathrm{MOE}_{d}$. Varios investigadores han determinado estos dos módulos de elasticidad en la madera, y el $\mathrm{MOE}_{e}$ en función del $\mathrm{MOE}_{d}$ ha sido muy bien correlacionado (Halabe et al.1997, Wang y Ko 1998, Tsehaye et al. 2000, Wang et al. 2000, Lindström et al. 2002).

Durante la última década, bastante atención ha recibido la aplicación de métodos acústicos para la evaluación mecánica, tanto de árboles en pie como en trozas con fines estructurales. Estas metodologías se basan en la propagación de ondas de esfuerzos que viajan a través de una porción del fuste de un árbol o a lo largo de una troza. Los métodos más utilizados son el tiempo de vuelo (TV) y la resonancia (Res) (Grabianowski et al. 2004). En el método de Tv, se controla el tiempo de viaje de una onda de esfuerzo entre dos sensores acústicos (un emisor y un receptor) ubicados a una distancia conocida (Wang et al. 2007a), y la velocidad se obtiene de la relación tiempo (t) / distancia (d). El método de Res permite la determinación directa de la velocidad de viaje de una onda de esfuerzo, de acuerdo a su frecuencia de resonancia en una distancia conocida (Andrews 2002). Estos métodos se utilizan en árboles en pie y trozos, para el caso de Tv, y sólo en trozos, para el método de Res.

Los métodos acústicos mencionados anteriormente, de bajo costo, permiten la evaluación no destructiva de los árboles en pie y son eficientes para la clasificación de la materia prima de los bosques (Huang et al. 2003). Además, estos métodos proporcionan información estratégica que puede ayudar a tomar decisiones de manejo y ambientales, en los tratamientos para los árboles individuales y rodales, mejorar las operaciones de raleo y cosecha, y asignar eficientemente los recursos madereros para la utilización óptima (Wang et al. 2007b).

Una aplicación exitosa de esta tecnología, ha involucrado cambios en la forma de manejo de las plantaciones. Recientes investigaciones muestran que el $\mathrm{MOE}_{d}$ de la madera juvenil de Pinus radiata puede ser manipulado por el manejo. En sitios individuales, se ha demostrado un aumento significativo y sustancial en el $\mathrm{MOE}_{d}$ mediante el aumento de la densidad de plantación inicial (DPI) del rodal, en ensayos de 11 (Lasserre et al. 2004, 2005) y 17 años (Waghorn et al. 2007a, b). Sin embargo, estos reportes muestran claramente la tendencia del $\mathrm{MOE}_{d}$ a igualarse, a medida que la DPI aumenta. Según Waghorn et al. (2007b) esto estaría indicando que, al margen de la DPI, el $\mathrm{MOE}_{d}$ de la periferia de los árboles converge con la edad, y por otro lado, el aumento de la edad 
y la acumulación de madera adulta podrían generar este cambio (Lasserre et al. 2009). Por estas razones, probablemente, Grabianowski et al. (2004), no encontró diferencias significativas del $\mathrm{MOE}_{d}$, en árboles en pie de Pinus radiata de 27 años de edad, creciendo en densidades de plantación de 100 y $625 \mathrm{arb} \cdot \mathrm{ha}^{-1}$, aunque, el ensayo evaluado no contempló tratamientos de DPI, ya que las densidades de $100 \mathrm{arb} \cdot h \mathrm{a}^{-1}$ se obtuvieron mediante la aplicación de raleos a los 11 y 13 años de edad.

En consecuencia, de acuerdo a los reportes de Lasserre et al. (2005) y Waghorn et al. (2007b), se podría pensar que, el $\mathrm{MOE}_{d}$ de plantaciones de Pinus radiata tienden a equilibrarse a una edad adulta, independiente de su DPI. Sin embargo, estas plantaciones corresponden a material joven, no superior a 20 años de edad, creciendo en Nueva Zelandia. Por lo tanto, la presente investigación tuvo como objetivo general, evaluar el efecto de la DPI en el $\mathrm{MOE}_{d}$ de la madera de un ensayo de Pinus radiata adulto, de 28 años de edad, creciendo en Chile. Específicamente, a) evaluar el efecto de la DPI en el estado de desarrollo de los árboles, densidad verde, velocidad acústica y en el $\mathrm{MOE}_{d}$ de árboles en pie y volteados (primera y segunda troza), b) relacionar el $\mathrm{MOE}_{d}$ de trozas versus $\mathrm{MOE}_{d}$ de árboles en pie, y c) relacionar los $\mathrm{MOE}_{d}$ con las variables dendrométricas diámetro a la altura del pecho (Dap) y esbeltez (Es).

\section{MATERIAL Y MÉTODO}

\section{Material y zona de estudio}

Se muestrearon árboles de Pinus radiata a la edad de 28 años creciendo en un ensayo de densidad de plantación inicial (DPI) ubicado en el predio Colicheu, $15 \mathrm{~km}$ al noreste de la ciudad de Cabrero,

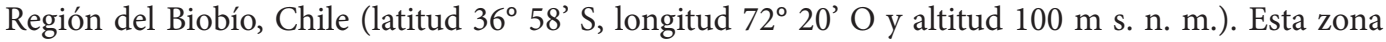
posee un clima templado cálido, con cuatro a cinco meses secos, y una precipitación promedio anual de $1.100 \mathrm{~mm}$, concentrada entre los meses de mayo y agosto (Fuenzalida 1971). Las DPI evaluadas de este ensayo, se muestran en la Tabla 1.

El ensayo fue instalado en el año 1980, en un suelo de la serie Arenales, caracterizados por ser moderadamente profundos, ricos en arena (superior al $93 \%$ ), pobres en arcilla, con bajo contenido de nitrógeno y de materia orgánica. Estos suelos se presentan en forma de llanos aluviales de baja pendiente $(2 \%)$ y poseen una alta tasa de infiltración y baja capacidad de campo (Carrasco et al. 1993).

El origen de las semillas fue desconocido para todo el rodal y al momento de la medición, los árboles de todos los tratamientos del ensayo presentaban una poda baja de 2 a $3 \mathrm{~m}$ de altura.

Tabla 1. Tratamientos de densidad de plantación inicial (DPI) en Pinus radiata.

\begin{tabular}{|c|c|c|}
\hline \multirow{2}{*}{$\begin{array}{c}\text { TRATAMIENTO } \\
\text { DPI } \\
\left(\text { árb } \cdot \mathrm{ha}^{-1}\right)\end{array}$} & $\begin{array}{c}\text { Arreglo } \\
(\mathrm{m})\end{array}$ & $\begin{array}{c}\text { DEspaciamiento } \\
\left(\mathrm{m}^{2}\right)\end{array}$ \\
\cline { 2 - 3 } 2500 & $2 \times 2$ & 4 \\
1667 & $2 \times 3$ & 6 \\
833 & $3 \times 4$ & 12 \\
625 & $4 \times 4$ & 16 \\
\hline
\end{tabular}

\section{Selección de árboles}

Se seleccionaron nueve árboles por tratamiento, de forma aleatoria, desde los estratos dominantes y codominantes, por ser los árboles más representativos de un rodal. No se ejecutó ningún raleo en 
alguna etapa previa del desarrollo de los árboles y, debido a la mortalidad natural en los tratamientos, la selección se realizó teniendo en consideración que cada unidad seleccionada debía estar rodeada por árboles vivos. Los árboles seleccionados se marcaron en terreno con un anillo de pintura blanca sobre el diámetro a la altura del pecho (Dap) y una línea vertical en el punto cardinal norte.

Antes de realizar las mediciones acústicas se midió el Dap, la altura total $(H t)$ y la calidad de los árboles (rectitud, estado fitosanitario y clasificación sociológica). Con esta información se determinó la sobrevivencia, el índice de sitio $^{3}$ y la Es. La sobrevivencia y el índice de sitio se determinaron sobre la base de la totalidad de árboles por tratamiento. En cambio, la Es se obtuvo sólo de los árboles muestra, y corresponde a la razón $H t / D a p\left(\mathrm{~m} \cdot \mathrm{m}^{-1}\right)$.

\section{Determinación del MOE $E_{\mathrm{d}}$ en árboles en pie}

En árboles en pie se midió el tiempo de vuelo $(T v)$ de una onda acústica entre dos puntos de distancia conocida $(d)$ y, mediante la relación $d / T v$, se obtuvo la velocidad de la onda acústica $(\mathrm{V})$. Con este valor, más la densidad verde $(r)$ de la troza 1 , se obtuvo el $\mathrm{MOE}_{d}$ mediante la expresión:

$$
\mathrm{MOE}_{\boldsymbol{d}}=\rho \mathrm{V}^{2}
$$

El Tv se midió con el instrumento portátil Tree Sonic, posicionando dos sensores acústicos en el fuste del árbol, a $1 \mathrm{~m}$ de distancia entre ellos, a partir de $0.8 \mathrm{~m}$ desde el piso (Figura 1a). Se realizaron dos mediciones para cada árbol, una en el punto cardinal norte del fuste (ya identificado con una línea vertical de color blanco) y la otra, en la dirección oeste. Estas mediciones se realizaron sin quitar la corteza y cada una de ellas es el resultado del promedio de tres lecturas de Tv sobre cada punto cardinal. Este mismo procedimiento, llamado método de "Tiempo de vuelo", ha sido usado por varios autores (Lasserre et al. 2005 y 2008, Waghorn et al. 2007b, Mora et al. 2009).

a)

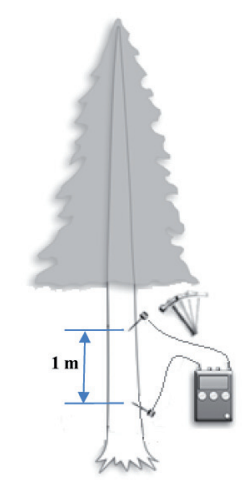

Tiempo de vuelo $(T v)$ ÁRBOL EN PIE b)

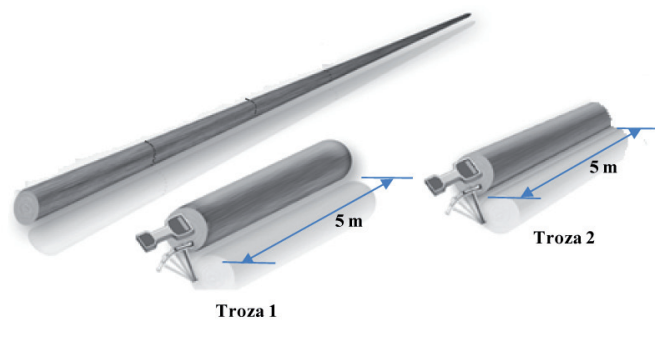

Resonancia ( $R e s)$

ÁRBOLVOLTEADO

Figura 1. Medición del Tv en árboles en pie (a) y de la $\mathrm{V}$ acústica en trozos (b).

(La figura no está a escala).

\section{Determinación del MOE $\mathrm{d}_{\mathrm{d}}$ en trozos}

Desde la base y a lo largo del fuste de cada árbol seleccionado se obtuvieron dos trozas de $5 \mathrm{~m}$ de longitud. En cada troza se midió directamente la $\mathrm{V}$ con el instrumento Hitman, herramienta que 
mide la V por el llamado método de "Resonancia" (Res). Esta velocidad se validó al menos tres veces en la cara con mayor diámetro de cada trozo (Figura 1b). Este valor y la $r$ de cada troza se ingresaron directamente a la Ecuación 1, para obtener el valor de $\mathrm{MOE}_{d}$ de las trozas.

\section{Determinación de la densidad actual}

Después de realizadas las mediciones acústicas en los árboles en pie y en las trozas de los árboles, se extrajeron tres rodelas ubicadas en el extremo de los trozos $(0,5$ y $10 \mathrm{~m})$ y una rodela en la posición del Dap, a $1.3 \mathrm{~m}$ del nivel del suelo. Cada rodela fue descortezada y pesada en terreno, luego etiquetada y, en bolsas de plástico selladas, transportadas al Laboratorio Silvotecnológico de la Madera (LSTM), en la Facultad de Ciencias Forestales de la Universidad de Concepción. En el laboratorio se midió su volumen por desplazamiento de agua, siguiendo las recomendaciones del método B de la norma americana ASTM D-2395. Para la determinación de la $\rho$ en las rodelas se utilizó la siguiente expresión:

$$
\rho=\mathrm{p} v / \mathrm{v} v
$$

Donde $\mathrm{p} v$ y $\mathrm{v} v$ es el peso y volumen verde actuales de la rodela, controlados en terreno y en laboratorio, respectivamente, razón por la cual a la $\rho$ también se le identifica como densidad actual, ya que puede variar con la estación del año y condiciones de cultivo de los árboles (Timber Advisory Notes 2005).

En esta investigación para efectos de análisis de esta variable, la $\rho$ correspondió al promedio de los valores de las rodelas a la altura de $0,1,3($ Dap ) y $5 \mathrm{~m}$, para la primera troza, y al promedio de las rodelas a los 5 y $10 \mathrm{~m}$ de altura, para la segunda troza.

Por otro lado, con la $\rho$ de la primera troza se calculó el $\mathrm{MOE}_{d}$ (por Tv) de los árboles en pie y, con la $\rho$ de la primera y segunda troza, se calculó el $\mathrm{MOE}_{d}$ (por Res) de la primera y segunda troza, respectivamente.

\section{Determinación del porcentaje de madera tardía}

El porcentaje de madera tardía se determinó en varillas de $1 \times 1 \mathrm{~cm}$ en espesor y ancho, respectivamente, tomadas de médula a corteza, en el punto cardinal norte de los fustes, a 2,5 $\mathrm{m}$ de altura. Las mediciones se realizaron con la tecnología proporcionada por SilviScan-2 (Evans 2005), en el laboratorio de FPInnovations - Paprican, Vancouver, Canadá. La tecnología SilviScan se compone de un escáner celular, un densitómetro de rayos $\mathrm{x}$ y un difractómetro de rayos $\mathrm{x}$, permitiendo el análisis de imágenes con el tamaño, tipo y distribución de células; la determinación de la densidad del material y la medición del ángulo de la microfibrilla.

\section{Análisis de datos}

Los efectos de la DPI en los parámetros de desarrollo de los árboles y en el $\mathrm{MOE}_{d}$ por $T v$ y por Res se determinaron mediante un análisis de varianza (andeva) y un test de comparación de medias de Tukey (con un nivel de significancia de 0.05). Todos los análisis se realizaron en SAS (SAS-InstituteInc. 2000).

Las relaciones entre el $\mathrm{MOE}_{d}$ por Tv y Res, $\mathrm{MOE}_{d}$ entre tipos de trozas, $\mathrm{MOE}_{d}$ con el Dap y Es, se realizaron a través de análisis de regresión simple. 


\section{RESULTADOS Y DISCUSIÓN}

\section{Estado de desarrollo de los árboles asociados a los tratamientos}

Se sabe que, la sobrevivencia es mayor a medida que la densidad de plantación inicial (DPI) disminuye. Los resultados muestran que esta tendencia no se encontró en los tratamientos analizados (Tabla 2), fundamentalmente, dado a que la sobrevivencia mostró estar directamente relacionada con el índice de sitio. Por lo tanto, la menor sobrevivencia encontrada en el tratamiento de 833 árb·ha ${ }^{-1}$, se debe al índice de sitio notablemente más bajo. Esto permite señalar que sitios de baja productividad no soportarían densidades tan altas.

La diferencia entre índices de sitio se manifiesta en los tratamientos de menor DPI. Primeramente este índice, baja fuertemente de 24 a $21.4 \mathrm{~m}$, desde el tratamiento de 1667 al de 833 árb ha ${ }^{-1}$,

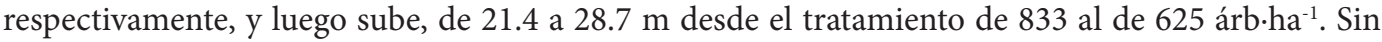
embargo, en suelos de arena no resulta sorprendente encontrar tanta variación en un área plana y reducida. Los suelos arenosos de la zona de estudio se caracterizan por ser altamente variables y presentar limitaciones en el crecimiento y desarrollo de las plantas, debido a la poca presencia de agua disponible (Carrasco y Millán 1990).

El efecto de la DPI en el Dap fue significativo $(p<0.05)$, como se muestra en la Tabla 2, donde el Dap aumentó a medida que la DPI disminuyó, llegando a ser un $62 \%$ mayor en el tratamiento de 625 árb $\cdot h a^{-1}$ con respecto al de 2500 árb $\cdot h a^{-1}$.

Tabla 2. Variables de estado (del bosque) y del tamaño de los árboles muestreados al momento de la medición (28 años de edad).

\begin{tabular}{|c|c|c|c|c|c|c|c|c|c|c|}
\hline \multirow{4}{*}{$\begin{array}{c}\text { TRAT. } \\
\text { DPI } \\
\left(\text { árb·ha }^{-1}\right) \\
\end{array}$} & \multicolumn{3}{|c|}{ Parámetros de desarrollo de los Tratamientos } & \multicolumn{7}{|c|}{ Caracteristicas dasométricas de la muestra } \\
\hline & \multirow{3}{*}{$\begin{array}{l}N \\
\text { rb.hat } \\
\end{array}$} & \multirow{3}{*}{\begin{tabular}{|c} 
Sobrevivencia \\
$(\%)$ \\
\end{tabular}} & \multirow{3}{*}{\begin{tabular}{|c} 
Indice de Sitio \\
(m) \\
\end{tabular}} & \multirow{3}{*}{$\begin{array}{c}n \\
\text { (árb) } \\
\end{array}$} & \multirow{2}{*}{\multicolumn{2}{|c|}{$\begin{array}{l}\text { Dap }(\mathrm{cm}) \\
p<0.0001 \\
\end{array}$}} & \multirow{2}{*}{\multicolumn{2}{|c|}{$\begin{array}{c}H t(\mathrm{~m}) \\
p<0.0001\end{array}$}} & \multirow{2}{*}{\multicolumn{2}{|c|}{\begin{tabular}{|l|}
$E s\left(\mathrm{~m} \cdot \mathrm{m}^{-1}\right)$ \\
$p<0.0001$ \\
\end{tabular}}} \\
\hline & & & & & & & & & & \\
\hline & & & & & $\begin{array}{c}\text { Dap (cm) } \\
(\mathbf{c m})\end{array}$ & Tukey & $\begin{array}{l}\boldsymbol{H t} \\
(\mathrm{m})\end{array}$ & Tukey & $\begin{array}{c}E s \\
\left(\mathbf{m} \cdot \mathbf{m}^{-1}\right)\end{array}$ & Tukey \\
\hline 2500 & 1749 & $70 \%$ & 24.4 & 9 & $23.9 \pm 2.4$ & A & $27.1 \pm 2.2$ & A & $113.9 \pm 7.36$ & A \\
\hline 1667 & 1136 & $68 \%$ & 24.0 & 9 & $27.4 \pm 2.6$ & $\mathrm{~B}$ & $28.4 \pm 2.5$ & A & $104.0 \pm 5.74$ & $\mathrm{~B}$ \\
\hline 833 & 520 & $62 \%$ & 21.4 & 9 & $31.6 \pm 2.4$ & $\mathrm{C}$ & $25.7 \pm 3.9$ & A & $81.4 \pm 9.42$ & $\mathrm{C}$ \\
\hline 625 & 541 & $87 \%$ & 28.7 & 9 & $38.3 \pm 4.1$ & $\mathrm{D}$ & $34.4 \pm 1.3$ & B & $90.4 \pm 8.31$ & D \\
\hline
\end{tabular}

$N$ corresponde a la cantidad de árboles vivos por hectárea presentes en el rodal al momento de la evaluación. $\boldsymbol{n}$ corresponde a la cantidad de árboles muestreados por tratamiento.

Cada valor mostrado de Dap, Ht y Es corresponde al promedio por tratamiento, seguido por la Desviación Estándar. Letras distintas indican diferencias significativas $(p<0.05)$.

Por otro parte, la $H t$ presentó un comportamiento totalmente ajeno a lo esperado, donde esta variable de estado no fue afectada estadísticamente por la DPI en los tres primeros tratamientos (2500, 1667 y 833 árb $\cdot$ ha $\left.^{-1}\right)$, como se puede apreciar en la Tabla 2. Sin embargo, la $H t$ del tratamiento de menor DPI fue estadísticamente diferente de los otros tres tratamientos de mayor DPI. Esto probablemente es consecuencia de la calidad del sitio, lo cual también se reflejó en los porcentajes de sobrevivencia, como fuera anteriormente mencionado.

En relación con la Es de los árboles, esta disminuyó a medida que la DPI se hizo menor, con diferencias estadísticamente significativas entre los cuatro tratamientos, como se muestra en la tabla 2. Sin embargo, la DPI de 833 árb·ha ${ }^{-1}$ presentó un menor valor que la DPI de 625 árb·ha ${ }^{-1}$. La razón de esta diferencia se debe a la baja $H t$ que presentaron los árboles muestreados para este tratamiento. 


\section{Comportamiento de la $\rho$}

De acuerdo a la Tabla 3, la $\rho$ aumentó con la disminución de la DPI de 2500 a 625 árb·ha ${ }^{-1}$, tanto para la primera como para la segunda troza, con diferencias significativas entre los tratamientos más densos (2500 y 1667 árb·ha ${ }^{-1}$ y el menos denso (625 árb·ha $\left.{ }^{-1}\right)$. Este aumento en la $\rho$ se puede deber a cambios en la micro estructura de la madera, relacionado con el tamaño de las traqueidas (Panshin y De Zeeuw 1980). Al respecto, Sandoval (2011), trabajando con los mismos tratamientos y árboles, determinó un aumento de la porosidad de la madera con la disminución de la DPI. Esto significa que, para un mismo volumen de madera, al disminuir la DPI la cantidad de agua aumenta (Ecuación 2).

Cabe también señalar que en cada tratamiento, la $\rho$ fue menor en la segunda troza con respecto a la primera. Sin embargo, sólo fue estadísticamente diferente para el tratamiento de 625 árb ha ${ }^{-1}$ $(p=0.0472)$.

Los resultados de la $r$ presentados en la tabla 3, no dejan de ser interesantes, dado a que varios autores, para determinar el $\mathrm{MOE}_{d}$, ya sea en trozas o en árboles en pie, asumen un valor de la $\rho$ constante, igual a $1000 \mathrm{Kg} / \mathrm{m}^{3}$ (Watt et al. 2009); o calculan el promedio de los valores obtenidos por árbol y lo usan como una $\rho$ constante para el rodal (Lasserre et al. 2005 y 2008, Waghorn et al. 2007b); o, simplemente, usan sólo la V ó V² para evaluar tratamientos silviculturales (Chauhan y Walker 2006, Grabionowski et al. 2006, Toulmin y Raymond 2007). Sin embargo, los valores de $\rho$ obtenidos en este estudio (Tabla 3), indican que dichas decisiones se deberían limitar sólo a los casos en que se tenga plena certeza que ésta no está afectada por los tratamientos silviculturales.

Tabla 3. Resultados de la $\rho$, medida en las trozas 1 y 2, y de la $\mathrm{V}$, en árboles en pie y trozas.

\begin{tabular}{|c|c|c|c|c|c|c|c|c|c|c|}
\hline \multicolumn{11}{|c|}{ 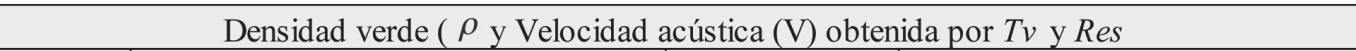 } \\
\hline \multirow{4}{*}{$\begin{array}{c}\text { TRAT. } \\
\text { DPI } \\
\left(\text { árb·ha }^{-1}\right)\end{array}$} & \multicolumn{4}{|c|}{$\rho$} & \multirow{2}{*}{\multicolumn{2}{|c|}{\begin{tabular}{|c|}
$\mathrm{V}$ por $T v$ \\
árb. en pie \\
\end{tabular}}} & \multicolumn{4}{|c|}{ V por Res } \\
\hline & \multicolumn{2}{|c|}{ árb. en pie y troza 1} & \multicolumn{2}{|c|}{ troza 2} & & & \multicolumn{2}{|c|}{ troza 1} & \multicolumn{2}{|c|}{ troza 2} \\
\hline & \multicolumn{2}{|c|}{$p=0.0006$} & \multicolumn{2}{|c|}{$p=0.0037$} & \multicolumn{2}{|c|}{$p<0.0010$} & \multicolumn{2}{|c|}{$p=0.0724$} & \multicolumn{2}{|c|}{$p=0.0432$} \\
\hline & $\begin{array}{l}\rho \\
\left(\mathbf{k g} \cdot \mathbf{m}^{-3}\right)\end{array}$ & Tukey & $\begin{array}{l}\rho \\
\left(\mathrm{kg} \cdot \mathrm{m}^{-3}\right)\end{array}$ & Tukey & $\begin{array}{c}\mathrm{V} \\
\left(\mathrm{km} \cdot \mathrm{s}^{-1}\right)\end{array}$ & Tukey & $\begin{array}{c}\mathrm{V} \\
\left(\mathrm{km} \cdot \mathrm{s}^{-1}\right)\end{array}$ & Tukey & $\begin{array}{c}\mathrm{V} \\
\left(\mathrm{km} \cdot \mathrm{s}^{-1}\right)\end{array}$ & Tukey \\
\hline 2500 & $0.82 \pm 0.06$ & A & $0.78 \pm 0.07$ & A & $3.76 \pm 0.30$ & A & $3.18 \pm 0.15$ & A & $3.36 \pm 0.15$ & A \\
\hline 1667 & $0.83 \pm 0.05$ & A B & $0.80 \pm 0.06$ & A B & $3.61 \pm 0.20$ & A & $3.11 \pm 0.18$ & A & $3.30 \pm 0.17$ & A B \\
\hline 833 & $0.87 \pm 0.03$ & $\mathrm{~B} \mathrm{C}$ & $0.85 \pm 0.04$ & $\mathrm{~B} \mathrm{C}$ & $3.67 \pm 0.17$ & A & $2.99 \pm 0.23$ & A & $3.14 \pm 0.17$ & B \\
\hline 625 & $0.91 \pm 0.04$ & $\mathrm{C}$ & $0.87 \pm 0.04$ & $\mathrm{C}$ & $4.04 \pm 0.19$ & B & $3.20 \pm 0.14$ & $\mathrm{~A}$ & $3.28 \pm 0.12$ & A B \\
\hline
\end{tabular}

Cada valor mostrado de $\rho$ y V corresponde al promedio por tratamiento, seguido por la Desviación Estándar. Letras distintas indican diferencias significativas $(p<0.05)$.

\section{Velocidad acústica de árboles en pie y trozas}

La velocidad acústica en los árboles en pie no fue influenciada por la DPI, para los tres tratamientos más densos (Tabla 3). Sin embargo, el tratamiento de menor DPI ( 625 árb·ha ${ }^{-1}$ ), muestra la velocidad más alta y estadísticamente diferente a los otros tres tratamientos.

Para el caso de los trozos (V obtenida por Res), en la troza 1, el tratamiento con DPI 625 árb ha ${ }^{-1}$ también presenta el mayor valor, pero sin diferencias significativas con los demás. En la troza 2 en cambio, la DPI 2500 árb·ha ${ }^{-1}$ es el tratamiento de mayor V, y estadísticamente diferente solo del tratamiento de 833 árb $\cdot$ ha $^{-1}$.

Las velocidades obtenidas por Tv fueron mayores que las obtenidas por Res, ya que en las primeras la trayectoria de vuelo entre dos puntos es en la madera adulta periférica, mientras que en la velocidad por Res, se está evaluando la sección transversal promedio ponderada, que incluye 
además la madera juvenil y la corteza (Grabianowski et al. 2006).

Así mismo, investigaciones anteriores muestran que velocidades medidas por $T v$ superan entre $9 \%$ y $30 \%$ a la velocidades medidas por Res (Andrews 2000 y 2003, Chauhan 2004, Chauhan y Walker 2006). Por consiguiente, los resultados presentados en la Tabla 3, son consistentes con los de dichas investigaciones. En efecto, los valores de $\mathrm{V}$ determinados por $\mathrm{Tv}$ presentados en dicha tabla, son un $18 \%, 16 \%, 23 \%$ y $26 \%$, mayores que los obtenidos por el método de Res, para las DPI de 2500, 1667, 833 y 625 árb·ha ${ }^{-1}$, respectivamente.

\section{$M O E_{d}$ en árboles en pie y en trozas}

El $\mathrm{MOE}_{d}$ de los árboles en pie (por Tv) no fue afectado por el aumento de la DPI en los tres tratamientos más densos $\left(2500,1667,833\right.$ árb $\left.\cdot h a^{-1}\right)$, aunque la diferencia la marcó el tratamiento de menor DPI (625 árb·ha-1 $)$, como se puede apreciar en Tabla 4.

Tabla 4. $\mathrm{MOE}_{d}$ de los árboles en pie y de la primera y segunda troza de los árboles volteados.

\begin{tabular}{|c|c|c|c|c|c|c|}
\hline \multicolumn{7}{|c|}{ MÓDULO DE ELASTICIDAD DINÁMICO SEGÚN MÉTODO DE Tv y Res } \\
\hline \multirow{2}{*}{ TRAT. } & \multirow{2}{*}{\multicolumn{2}{|c|}{$\frac{T v}{\text { árboles en pié }}$}} & \multicolumn{4}{|c|}{ Res } \\
\hline & & & & & & \\
\hline \multirow[b]{2}{*}{$\begin{array}{c}\text { DPI } \\
\left(\text { árb·ha }^{-1}\right)\end{array}$} & \multicolumn{2}{|c|}{$p<0.0001$} & \multicolumn{2}{|c|}{$p=0.0133$} & \multicolumn{2}{|c|}{$p=0.1164$} \\
\hline & $\begin{array}{c}\mathrm{MOE}_{d} \\
(\mathrm{GPa})\end{array}$ & Tukey & $\begin{array}{c}\mathrm{MOE}_{d} \\
(\mathrm{GPa})\end{array}$ & Tukey & $\begin{array}{r}\mathrm{MOE}_{d} \\
(\mathrm{GPa})\end{array}$ & Tukey \\
\hline 2500 & $11.7 \pm 2.4$ & A & $8.3 \pm 1.2$ & A B & $8.7 \pm 1.3$ & A \\
\hline 1667 & $10.7 \pm 1.3$ & A & $8.0 \pm 1.1$ & A & $8.5 \pm 1.0$ & A \\
\hline 833 & $11.7 \pm 1.1$ & A & $7.8 \pm 1.1$ & A & $8.3 \pm 0.8$ & A \\
\hline 625 & $15.1 \pm 1.8$ & B & $9.4 \pm 1.0$ & B & $9.4 \pm 0.8$ & A \\
\hline
\end{tabular}

Cada valor de $\mathrm{MOE}_{d}$ mostrado corresponde al promedio por tratamiento, seguido por la Desviación Estándar. Letras distintas indican diferencias significativas $(p<0.05)$.

En la troza 1 el $\mathrm{MOE}_{d}$ no se diferenció estadísticamente en los tres tratamientos más densos (2500, 1667, 833 árb·ha $\left.^{-1}\right)$, como se muestra en Tabla 4, pero mostró una tendencia a decrecer con la disminución de la DPI. Sin embargo, en el tratamiento de menor DPI esta tendencia se rompe, siendo el $\mathrm{MOE}_{d}$ de este tratamiento, más alto y diferente estadísticamente a los tratamientos de 833 y 1667 árb·ha ${ }^{-1}$.

Con respecto a la troza 2, se encontró que estadísticamente, el $\mathrm{MOE}_{d}$, no difiere entre tratamientos por efecto del aumento o disminución de la DPI (Tabla 4). Sin embargo, el $\mathrm{MOE}_{d}$ de la troza 2 tuvo el mismo comportamiento que en la troza 1.

Otros investigadores han encontrado un aumento del $\mathrm{MOE}_{e}$ y $\mathrm{MOE}_{d}$ con el aumento de la densidad del rodal para diferentes especies. Wang y Ko (1998) reportan un aumento del $\mathrm{MOE}_{e}$ y $\mathrm{MOE}_{d}$ con el aumento de la densidad del rodal en un ensayo de DPI de 41 años de edad, en Cryptomeria japonica. Los mismos resultados son reportados por Chuang y Wang (2001) para un ensayo con Cryptomeria japonica de 47 años de edad, y Wang et al. (2001) para Tsuga heterophylla y Picea sitchensis, de 38 a 70 años de edad, en un ensayo de cuatro intensidades de raleo. Investigaciones referidas a la especie Pinus radiata de 11 y 17 años de edad, realizadas por Lasserre et al. (2005) y por Waghorn et al. (2007b), respectivamente, reportan también un aumento del $\mathrm{MOE}_{d}$ con el aumento de la DPI del 
rodal. Sin embargo, Soto et al. (datos sin publicar) encontraron un comportamiento contrario a los descritos anteriormente, en un ensayo de poda y raleo de Pinus radiata de 32 años, creciendo en un suelo arcilloso, cercano a la ciudad de Mulchén, Región del Biobío, donde en tratamientos con un solo raleo a los ocho años, con densidades finales de 200, 400, 600 y 800 árb $\cdot h^{-1}$, el $\mathrm{MOE}_{d}$ por Tv creció a medida que disminuyó la densidad residual del rodal, de 15, 4 a 19,1 GPa.

Aunque de acuerdo al párrafo anterior, el efecto de la DPI en el $\mathrm{MOE}_{d}$ es contradictorio para la especie Pinus radiata, en esta investigación, con el incremento de la DPI, las características dasométricas de los árboles aumentaron y/o disminuyeron (Tabla 2) y el $\mathrm{MOE}_{d}$, tanto para árboles en pie como para las trozas, no mostró un comportamiento definido de aumentar o disminuir. Esto se explica por el efecto que tiene la DPI en el comportamiento de las variables $r$ y V (Tabla 3), que componen la Ecuación 1, para determinar el $\mathrm{MOE}_{d}$. En este caso, con la disminución de la DPI incrementó la $r$, tanto para la troza 1 como para la troza 2, pero la V, obtenida por Tv y Res, no fue afectada claramente por la DPI. Por lo tanto, el producto de $r$ con $\mathrm{V}^{2}$, entregó un $\mathrm{MOE}_{d}$ por Tv casi constante para los tratamientos de 2500, 1667 y 833 árb·ha ${ }^{-1}$, y un $\mathrm{MOE}_{d}$ por Res, tanto para la troza 1 como para la troza 2, levemente decreciente con la disminución de la DPI en estos mismos tratamientos, pero sin diferencias significativas entre sí. Sin embargo, el tratamiento de 625 árb·ha ${ }^{-1}$ presentó un $\mathrm{MOE}_{d}$, por Tv y Res, notablemente mayor al resto y similar entre las trozas 1 y 2 de dicho tratamiento.

Con respecto al $\mathrm{MOE}_{d}$ obtenido para las trozas, siempre el $\mathrm{MOE}_{d}$ de la segunda troza fue mayor que el de la primera, como se puede comparar en la Tabla 4. Xu y Walker (2004), trabajando con Pinus radiata de 27 años, reportan los mismos resultados. Ellos atribuyen esta diferencia al efecto del ángulo de la microfibrilla (AMF) en la rigidez de la madera. En este ensayo, el AMF fue medido de médula a corteza (sobre 23.000 traqueidas) por Araya (2010), en los mismos árboles usados en esta investigación, a 0,5 y $10 \mathrm{~m}$ de altura. Los resultados mostraron, que para los cuatro tratamientos, siempre el AMF disminuyó desde la base hasta los $10 \mathrm{~m}$ de altura en los fustes. Por lo tanto, la suposición de Xu y Walker (2004) tendría asidero.

\section{$M O E_{\mathrm{d}}$ en trozas versus $M O E_{\mathrm{d}}$ de árboles en pie}

$\mathrm{El} \mathrm{MOE}$ de la troza 1 y 2 se correlacionó positivamente con el $\mathrm{MOE}_{d}$ de los árboles en pie, como se muestra en la Figura 2, con ambas relaciones significantes al 1\% del nivel de confianza.

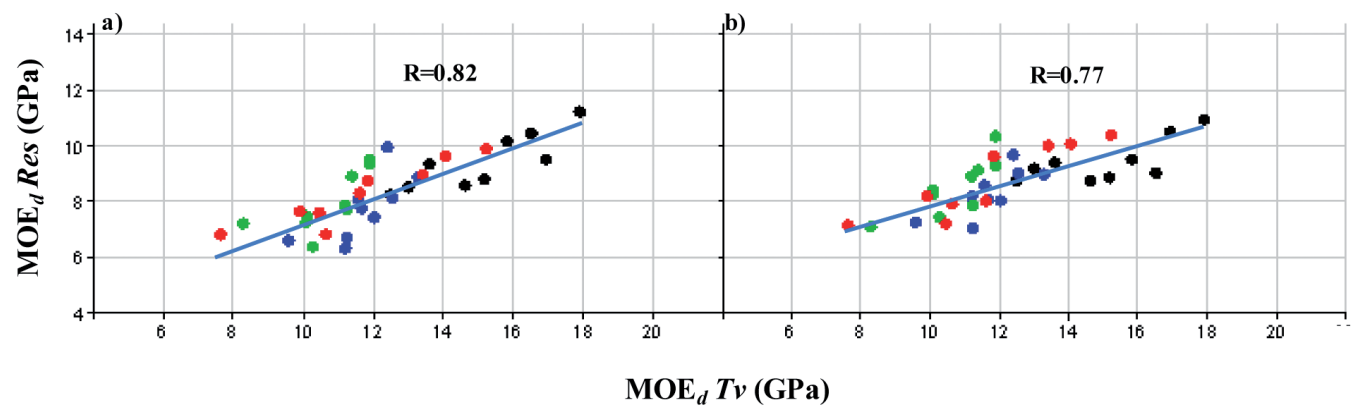

Figura 2. Relación entre el $\mathrm{MOE}_{d}$ por $T v$ y el $\mathrm{MOE}_{d}$ por Res, para la troza 1 (a) y la troza 2 (b), según DPI (•: 2500; ๑: 1667; •: 833 y •: 625).

Estos resultados son coincidentes con otras investigaciones que reportan un alto grado de correlación entre el $\mathrm{MOE}_{d}$ por Tv para árboles en pie y el $\mathrm{MOE}_{d}$ por Res para trozas basales de $5 \mathrm{~m}$ (Chauhan y Walker 2006, Mora et al. 2009). Sin embargo, en la literatura revisada no se encontraron investigaciones que relacionen el $\mathrm{MOE}_{d}$ por $T v$ para árboles en pie con el $\mathrm{MOE}_{d}$ por Res para la 
segunda troza, por lo tanto, este resultado toma importancia cuando, por el manejo silvicultural dado al bosque, el objetivo de la primera troza no es con fines estructurales.

\section{Relación del MOE ${ }_{\mathrm{d}}$ de árboles en pie y de la troza 1 con el Dap}

El Dap presentó una relación positiva con el $\mathrm{MOE}_{d}$ de los árboles en pie (Figura 3a), sin embargo sólo explicó un $31 \%$ de su variación. La relación con el $\mathrm{MOE}_{d}$ de la troza 1 también fue positiva (Figura 3b), pero sólo un 9\% de esta variación fue explicada por el Dap.

Este aumento del $\mathrm{MOE}_{d}$ a medida que crece el Dap, tanto para árboles en pie como para la troza 1 (Figura 3 a y b), es contrario a los resultados presentados en diversas investigaciones. Varios estudios reportan un aumento del $\mathrm{MOE}_{d}$ con el aumento de la densidad del rodal y, asocian este aumento, a reducciones en el diámetro del fuste (Zhang 1995, Zhang et al. 2002, Lasserre et al. 2005, Chauhan y Walker 2006). Zhang (1995) observó que dentro de un rodal los árboles más grandes, resultantes de altas tasas de crecimiento, por lo general, producen madera de baja rigidez. Chauhan y Walker (2006) sostienen que para árboles de la misma edad, individuos de crecimiento lento tienden a tener una alta rigidez en la periferia por el efecto de la fuerza del viento y el peso de la copa.

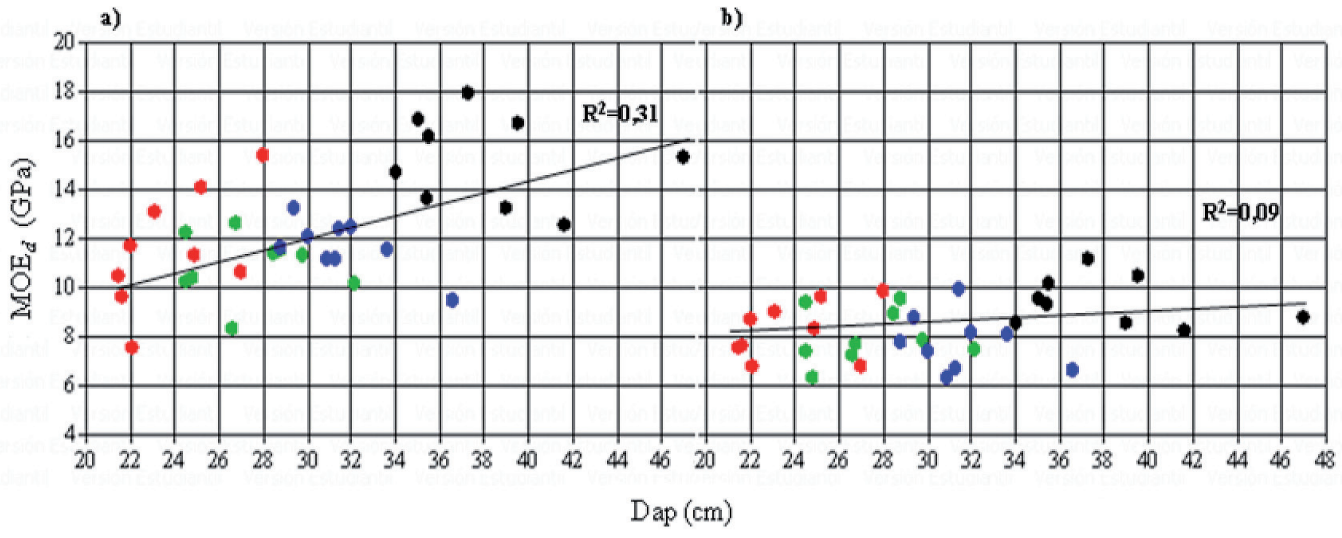

Figura 3. Relación entre el Dap y el $\mathrm{MOE}_{d}$, según el método de Tv para árboles en pie (a) y el

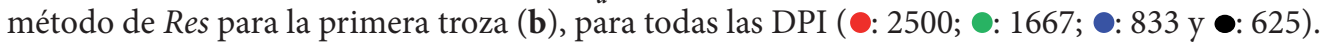

Dickson et al. (2004) relacionaron el Dap de Pinus radiata de 30 años con el $\mathrm{MOE}_{e}$ de piezas de madera de tamaño estructural, de madera juvenil (MJ) y madera adulta (MA). Estos autores concluyen que el Dap, como una medida del tamaño del árbol, influye en el $\mathrm{MOE}_{e}$ del trozo sólo cuando se relaciona con la MJ y MA, por separado. Sin embargo, estas relaciones siguen siendo negativas, es decir, con el aumento del Dap disminuye el $\mathrm{MOE}_{e}$, no obstante, ameritan una validación de los resultados del presente estudio a través de la determinación del $\mathrm{MOE}_{e}$ por tipo de madera.

Por otra parte, Lasserre et al. (2005), indican como posible explicación de esta relación inversa entre el Dap y el $\mathrm{MOE}_{d^{\prime}}$ la variación en el porcentaje de madera tardía entre los árboles de diámetros altos y bajos. Chuang y Wang (2001) informan que árboles de mayor diámetro tienen un menor porcentaje de madera tardía y menor densidad, que árboles con un diámetro más bajo. Por consiguiente, dado que las fibras de madera tardía son más rígidas que las de madera temprana (Cramer et al. 2005), la baja incidencia de la madera tardía en los árboles más grandes podría explicar la baja rigidez observada dentro de estos árboles. 

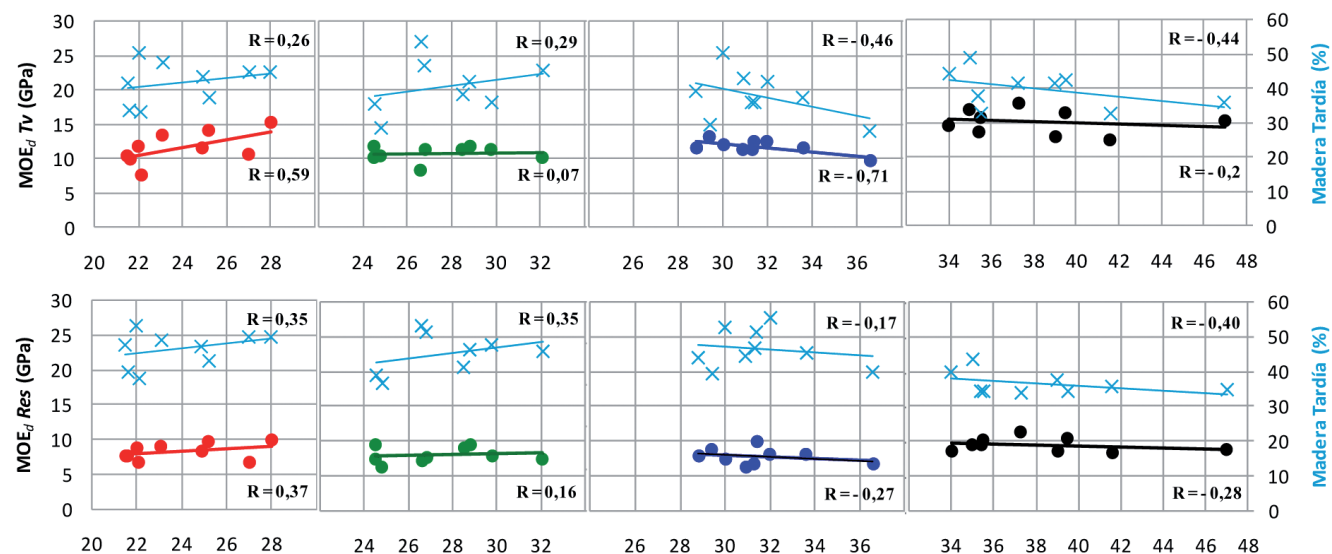

Dap (cm)

Figura 4. Relación del Dap con el $\mathrm{MOE}_{d}$ (por Tv para árboles en pie y por Res para la troza 1) y el porcentaje de madera tardía, para cada una de las DPI (•:2500;•: 1667;•: 833 y •: 625).

En la Figura 4 se muestra por separado, para cada DPI, la relación entre el Dap y el $\mathrm{MOE}_{d}$ por $T v$ y la relación entre el Dap y el $\mathrm{MOE}_{d}$ por Res. En ambos casos, en el eje ubicado a la derecha de la figura, se incorporó el porcentaje de madera tardía. Para relacionar con el $\mathrm{MOE}_{d}$ por Tv, el porcentaje de madera tardía se promedió para los $5 \mathrm{~cm}$ de la periferia del fuste, en el punto cardinal norte. En cambio, para correlacionar con el $\mathrm{MOE}_{d}$ por Res, el porcentaje de madera tardía se promedió para todo el radio norte de la sección transversal del fuste. En esta figura se puede observar que en general el $\mathrm{MOE}_{d}$ y el porcentaje de madera tardía de los dos tratamientos de mayor DPI (2500 y $1667 \mathrm{arb} \cdot \mathrm{ha}^{-1}$ ) presentaron una relación positiva con el Dap, es decir, el $\mathrm{MOE}_{d}$ (por Tv y Res) y el porcentaje de madera tardía, aumentaron a medida que aumentó el Dap, aunque con una respuesta muy débil por parte del $\mathrm{MOE}_{d}$ del tratamiento de $1667 \mathrm{arb} \cdot \mathrm{ha}^{-1}$. Sin embargo, para los dos tratamientos de menor DPI (833 y $625 \mathrm{arb} \cdot h \mathrm{a}^{-1}$ ) esta relación con el $\mathrm{MOE}_{d}$ (por Tv y Res) y con el porcentaje de madera tardía se hizo inversa, disminuyendo ambas variables a medida que aumentó el Dap.

Por lo tanto, en cada tratamiento el porcentaje de madera tardía estaría explicando el comportamiento del $\mathrm{MOE}_{d}$, por Tv y por Res, a medida que crece el Dap (Figura 4). Así, para los tratamientos menos densos, este comportamiento inverso al tamaño de los árboles, se explicaría por lo señalado por Chuang y Wang (2001), quienes sostienen que en rodales de igual edad, árboles de rápido crecimiento, y por ende de mayores Dap y anchos anillos de crecimiento, tendrán un menor porcentaje de madera tardía.

En consecuencia, las relaciones a nivel de tratamientos, mostradas en la Figura 4, permiten conceptuar el vínculo entre el Dapy el $\mathrm{MOE}_{d}$ por $T v$ y por Res (Figura 3 a y b), como una "pseudo relación". Por otra parte, por la restricción de agua disponible (Carrasco et al. 1993) y veranos secos presentes en el área estudiada (Fuenzalida 1971), los árboles de los tratamientos con alta DPI pueden haber sido más afectados durante el verano que aquellos de baja DPI, dejando de crecer antes debido a la falta de agua.

\section{Relación entre el MOE $\mathrm{d}_{\mathrm{d}}$ de árboles en pie y la Es}

La Es observada en este estudio (Tabla 2), en general muestra un descenso a nivel de promedios, a medida que disminuye la DPI. En la Figura 5 se puede observar que la relación entre la $E s$ y el $\mathrm{MOE}_{d}$ fue levemente al descenso, cuando se consideraron todos los tratamientos. Sin embargo, a nivel de 
DPI, resultaron diversas tendencias asociadas a los tratamientos.

Waghorn et al. (2007b), sostienen que la Es puede ser más importante que el diámetro del fuste en la regulación del $\mathrm{MOE}_{d}$, particularmente a altas densidades de rodal. En la especie Pinus radiata, dado a que es una especie exigente en luz, esto significa, que al crecer bajo competencia, prioriza un rápido crecimiento en altura, a expensas del crecimiento en diámetro, para así, asegurar no ser dominados por árboles vecinos.

Así, varios estudios informan sobre el efecto de la Es del fuste en el $\mathrm{MOE}_{d}$ medido en ensayos de espaciamiento, tanto para Pinus radiata (Waghorn et al. 2007b, Lasserre et al. 2008) como para Pinus taeda (Roth et al. 2007). Todos estos autores reportan relaciones positivas entre la Es y el $\mathrm{MOE}_{d}$, a medida que aumenta la DPI. Sin embargo, estos reportes se basan en edades entre 11 y 17 años para Pinus radiata y de 6 años para Pinus taeda.

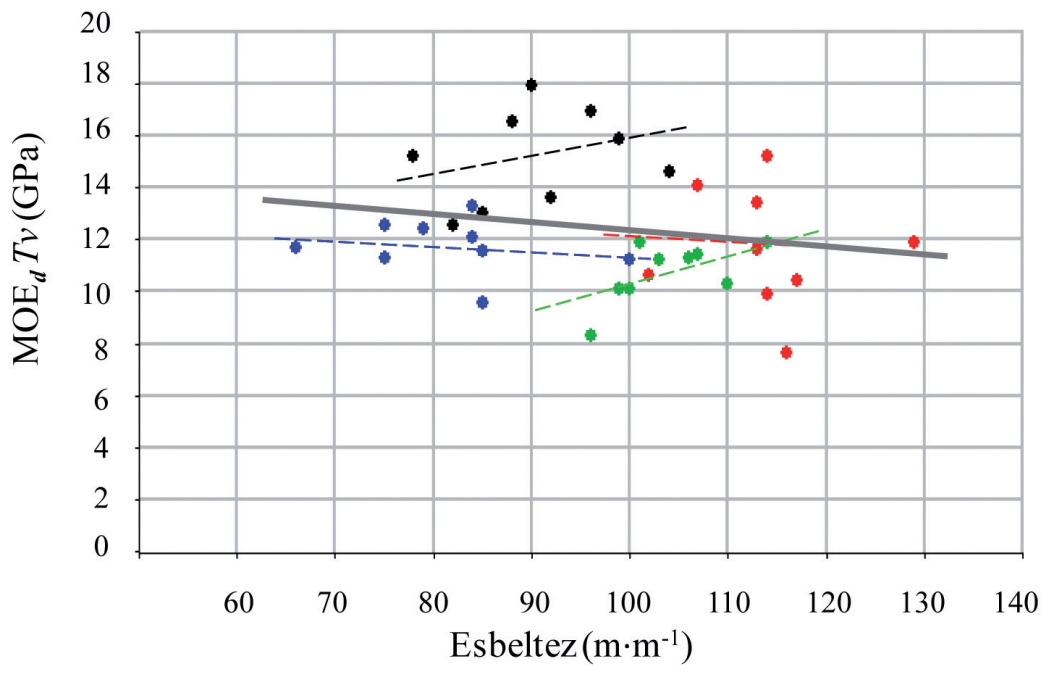

Figura 5. Relación entre la $E s(H t / D a p)$ y el $\mathrm{MOE}_{d}$ obtenido por $T v$, para árboles en pie, para cada DPI $(\bullet: 2500 ; \bullet: 1667 ; \bullet: 833$ y $\bullet: 625)$.

Waghorn et al. (2007b) y Lasserre et al. (2008) sostienen que la estrecha relación positiva observada entre la Es del fuste y el $\mathrm{MOE}_{d}$ ocurre porque árboles que crecen en rodales muy densos, con una alta $E s$, requieren alto MOE para mitigar la posibilidad de quebrarse a través de su mismo peso. Bajo este contexto se puede pensar que este efecto ocurre hasta que los árboles alcanzan su estabilidad, o el AMF tiende a estabilizarse, lo que explicaría el efecto poco claro observado en los resultados del presente estudio para Pinus radiata de 28 años (Figura 5).

Por otra parte, varios autores puntualizan que, además de la Es del fuste, la altura del comienzo de la copa viva de los árboles también se relaciona positivamente con el $\mathrm{MOE}_{d}$ y varía con la DPI del rodal (Waghorn et al.2007b, Mason 2006, Watt et al. 2006 y 2009), aunque en este estudio, dicha variable no se consideró.

\section{CONCLUSIONES}

Basado en los resultados obtenidos para este estudio de Pinus radiata creciendo en un suelo arenoso, se concluyó lo siguiente: 
El Dap y la Es resultaron afectadas por todas las DPI. Con la reducción de la DPI, el Dap aumentó y la Es disminuyó. Sin embargo, la Ht del tratamiento de menor DPI, resultó diferente de los otros tres tratamientos de mayor DPI.

La $\rho$ aumentó con la disminución de la DPI de 2500 a 625 árb·ha ${ }^{-1}$, pero, para una misma DPI, la $\rho$ fue menor en la segunda troza, con respecto a la primera.

En general, la V no fue influenciada por la DPI, sin embargo, el tratamiento de menor DPI (625 árb $\cdot h a^{-1}$ ) mostró la mayor $\mathrm{V}$, tanto para árboles en pie como para la primera troza.

El MOE ${ }_{d}$, por Tv y por Res, no fue afectado por la DPI en los tres tratamientos más densos (2500, 1667 y 833 árb·ha $\left.{ }^{-1}\right)$. Sin embargo, en el tratamiento menos denso (625 árb·ha ${ }^{-1}$ ) resultó el mayor $\mathrm{MOE}_{d}$, tanto por $T v$ como por Res, aunque similar entre las trozas 1 y 2.

El $\mathrm{MOE}_{d}$ de la troza 1 y 2 se correlacionó positivamente con el $\mathrm{MOE}_{d}$ de los árboles en pie.

La relación entre el Dap y el $\mathrm{MOE}_{d}$, por Tv y por Res, fue positiva para los tratamientos de mayor DPI (2500 y 1667 árb ha $^{-1}$ y negativa para los de menor DPI (833 y 625 árb·ha ${ }^{-1}$ ). Este comportamiento fue explicado por la misma variación experimentada por el porcentaje de madera tardía en función del Dap.

La relación entre la Es y el $\mathrm{MOE}_{d}$ por $T v$ fue negativa, aunque a nivel de tratamientos mostró diversas tendencias.

\section{BIBLIOGRAFÍA}

Andrews, M. 2000. Wherewe are withsonics? In: Workshop 2000. Capturing the Benefits of Forestry Research: Putting Ideas to Work. Wood Technology Research Centre, University of Canterbury. pp. 57-61.

Andrews, M. 2002. Wood quality measurement - son et lumiere. New Zealand Journal Forestry 47(3): 19-21.

Andrews, M. 2003. Which acoustic speed? In: Beall, F.C. (Editor). Proceedings of the 13th International Symposium on Nondestructive Testing of Wood, August 19-21, 2002. University of California, Richmond. pp. 159-165.

Araya, D. 2010. Efecto del espaciamiento inicial de plantación sobre el ángulo microfibrilar en Pinus radiata D. Don de 28 años de edad. Tesis Ing. Forest. Universidad de Concepción, Chile. 34 p.

ASTM. American Society for Testing and Materials. 1998. Standard Test Methods for Specific Gravity of Wood-Based Materials . ASTM D-2395. Annual Book of ASTM Standards. Section 4, Construction vol 0410 Wood. Easton. 666 p.

Carrasco, P.; Millán, J. 1990. Proyecto Suelos Forestales de la VIII Región. Informe Final. Convenio Universidad de Concepción y Ministerio de Agricultura, Fondos de Investigación Agropecuaria (F.I.A.). Chillán. 127 p.

Carrasco, P.; Millán, J.; Peña, L. 1993. Suelos de la cuenca del rio Bíobío. Características y problemas de uso. Gestión de recursos hídricos de la cuenca del rio Bíobío y del área costera adyacente. Serie Análisis Territorial. Ed. Universitaria de Concepción. 107 p. 
Chauhan, S. 2004. Selecting and/ or processing Wood according to its processing characteristics. PhD Thesis, University of Canterbury, Christchurch, New Zealand. 206 p.

Chauhan, S.; Walker, J. 2006. Variations in acoustic velocity and density with age, and their interrelation ships in radiata pine. Forest Ecology and Management 229: 388-394.

Chuang, S.; Wang, S. 2001. Evaluation of standing tree quality of Japanese cedar grown with different spacing using stress-wave and ultrasonic-wave method. Journal of Wood Science 47: 245-253.

Cramer, S.; Kretschmann, D.; Lakes, R.; Schmidt, T. 2005. Earlywood and latewood elastic properties in loblolly pine. Holzforschung 59: 531-538.

Dickson, R.; Mathesona, A.C.; Joe, B.; Ilic, J.; Owen, J.V. 2004. Acoustic Segregation of Pinus radiata logs for sawmilling. New Zealand Journal of Forestry Science 34(2): 175-189.

Evans, R. 2005. Wood stiffness by x-ray diffractometry. In: Stokke D. and L. Groom. (Editores). Workshop 2003. Characterisation of the cellulosic cell wall, Chapter 11. Grand Lake, Colorado, USA. pp. 1-7.

Fuenzalida, H. 1971. Clima: Geografía económica de Chile. Texto refundido. Corporación de Fomento de la producción. Santiago, Chile. pp. 99-152.

Grabianowski, M.; Manley, B.; Walker, J. 2004. Impact of stoking and exposure on outer wood acoustic properties of Pinus radiata in Eyrewell Forest. New Zealand Journal of Forestry 49(2): 13-17.

Grabianowski, M.; Manley, B.; Walker, J. 2006. Acoustic measurements on standing trees, logs and Green lumber. Wood Science and Technology 40: 205-216.

Halabe, U.B.; Bidigalu, G.M.; GangaRao, H.V.S.; Ross, R. 1997. Nondestructive evaluation of Green Wood using stress wave and transverse vibration techniques. Materials Evaluation 55(9): 1013-1018.

Huang, C.; Lindstrom, H.; Nakada, R.; Ralston, J. 2003. Cell wall structure and Wood properties determinated by acoustic- a selective review. Holz als Roh-undWerkstoff 61: 321-335.

Lasserre, J.P.; Mason, E.; Watt. M. 2004. The influence of initial stocking on corewood stiffness in a clonal experiment of 11-year-old Pinus radiata (D. Don). New Zealand Journal Forestry 49: 1823.

Lasserre, J.P.; Mason, E.; Watt, M. 2005. The effects of genotype and spacing on Pinus radiata (D. Don) corewood stiffness in an 11-year old experiment. Forest Ecology and Management 205: 375-383.

Lasserre, J.P.; Mason, E.; Watt, M. 2008. Influence of the main and interactive effects of site, stand density and clone on Pinus radiata D. Don corewood modulus of elasticity. Forest Ecology and Management 255: 3455-3459.

Lasserre, J.P.; Mason, E.; Watt, M.; Moore, J. 2009. Influence of initial planting spacing and genotype on micro fibril angle, Wood density, fibre properties and modulus of elasticity in Pinus radiata D. Don corewood. Forest Ecology and Management 258: 1924-1931.

Lindström, H.; Harris, P.; Nakada, R. 2002. Methods for measuring stiffness of Young trees. Holz als Roh-und Werkstoff 60: 165-174. 
Mason, E. 2006. Interactions between influences of genotype and grass competition on growth and wood stiffness of juvenile radiata pine in a summer-dry environment. Canadian Journal of Forest Research 36(10): 2454-2463.

Mora, C.R.; Schimleck, L.R.; Isik, F.; Mahon Jr., J.M.; Clark III, A.; Daniels, R.F. 2009. Relationships between acoustic variables and different measures of stiffness in standing Pinus taeda trees. Canadian Journal of Forest Research 39: 1421-1429.

Panshin, A.J.; De Zeeuw, C. 1980. Text book of Wood Technology. 4th edition. McGraw-Hill BookCo. New York. 722 p.

Roth, B.; Li, X.; Huber, D.; Peter, G. 2007. Effects of management intensity, genetic sand planting density on Wood stiffness in a plantation of juvenile loblolly pine in the south eastern USA. Forest Ecology and Management 246: 155-162.

Sandoval, L. 2011. Efecto del espaciamiento inicial en la densidad de la pared celular y la porosidad de la madera de Pinus radiata D. Don. Tesis Ing. Forest. Universidad de Concepción, Chile. 54 p.

Soto, L.; Valenzuela, L.; Lasserre, J.P. Módulo de elasticidad de árboles en pie de Pinus radiata creciendo a distintas densidades residuales de raleo. Sin publicar.

SAS Institute Inc. 2000. SAS/STAT User's Guide: Version 8, vol. 1-3. SAS Institute Inc., Cary, North Carolina, p. 3884.

Timber Advisory Notes. 2005. Background Information [en línea]. Disponible en <http://www. agric.wa.gov.au/PC_92548.html?s=0 > [consultado en marzo de 2012].

Toulmin, M. J.; Raymond, C.A. 2007. Developing a sampling strategy for measuring acoustic velocity in standing Pinus radiata using the Tree Tap time off light tool. New Zealand Journal of Forestry Science 37(1): 96-111.

Tsehaye, A.; Buchanan, A.H.; Walker, J. 2000. Sorting of logs using acoustics. Wood Science and Technology 34(4): 337-344.

Valenzuela, L.; Nakayama, Y. 1991a. The bending work of radiata pine grown in Chile. Mokuzai Gakkaishi 37(5): 396-404.

Valenzuela, L.; Nakayama, Y. 1991b. Predicting MOR of radiata pine grown in Chile, efficiency of some nondestructive parameters. Mokuzai Kogyo 46(5): 213-219.

Waghorn, M.J.; Mason, E.; Watt. M. 2007a. Influence of initial stand density and genotype on longitudinal variation in modulus of elasticity for 17-year-old Pinus radiata. Forest Ecology and Management 252: 67-72.

Waghorn, M.J.; Watt, M.; Mason, E. 2007b. Influence of tree morphology, genetics, and initial stand density on outer Wood modulus of elasticity of 17-year-old Pinus radiata. Forest Ecology and Management 244: 86-92.

Walford, G. 1985. The machanical Properties of New Zealand-grown Radiata Pine for Export to Australia. FRI Bulletin $N^{\circ}$ 93. Forest Research Institute, New Zealand Forest Service. 
Wang, S.Y.; Ko, C.Y. 1998. Dynamic modulus of elasticity and bending properties of large beams of Taiwan-grown Japanese cedar from different plantation spacing sites. Journal of Wood Science 44: $62-68$.

Wang, X.; Ross, R.; McClellan, M.; Barbour, R.; Erickson, J.; Forsman, J.; McGinnis, G. 2000. Strength and stiffness assessment of standing trees using a nondestructive stress wave technique. Forest Products Laboratory. Research Paper FPL-RP-585. 9 p.

Wang, X.; Ross, R.; McClellan, M.; Barbour, R.; Erickson, J.; Forsman, J.; McGinnis. G. 2001. Nondestructive evaluation of standing trees with a stress wave method. Wood and Fiber Science 33(4): 522-533.

Wang, X.; Ross, R.; Carter, P. 2007a. Acoustic evaluation of Wood quality in standing trees. Part 1. Acoustic wave behavior. Wood and Fiber Science 39(1): 28-38.

Wang, X.; Carter, P.; Ross, R.; Brashaw, B. 2007b. Acoustic assessment of wood quality of raw forest materials- a path to increased profitability. Forest Products Journal 57(5): 6-14.

Watt, M.; Moore, J.; Facon, J.F.; Downes, G.; Clinton, P.; Coker, G.; Davis, M.; Simcock, R.; Parfitt, R.; Dando, J.; Mason, E.; Bown, H. 2006. Modelling the influence of stand structural, edaphic and climatic influences on Pinus radiata dynamic modulus of elasticity. Forest Ecology and Management 229: 136-144.

Watt, M., Clinton, P.; Parfitt, R.; Ross, C.; Coker, G. 2009. Modelling the influence of site and weed competition on juvenile modulus of elasticity in Pinus radiata across broad environmental gradients. Forest Ecology and Management 258: 1479-1488.

Xu, P.; Walker, J. 2004. Stiffness gradients in Pinus radiata trees. Wood Science Technology 8:1-9.

Zhang, S.Y. 1995. Effect of growth rate on wood specific gravity and selected mechanical properties in individual species from distinct wood categories. Wood Science and Technology 29(6): 451-466.

Zhang, S.Y.; Chauret, G.; Ren, H.Q.; Desjardins, R. 2002. Impact of initial spacing on plantation Black spruce lumber grade yield, bending properties and MSR yield. Wood and Fiber Science 34(3): $60-475$. 